\title{
CEMR: Congestion evasion Multicast Routing to Maximize Throughput for Mobile Ad hoc Networks
}

\author{
Sailaja Chinege ${ }^{1,}$ P. Prasanna Murali Krishna ${ }^{2}$ \\ ${ }^{\text {I}}$ (Sailaja Chinege is currently doing her M.Tech at Dr.Samuel George Institute of Engineering and Technology, \\ Markapur, AP, India \\ ${ }_{2}^{2}$ (P.Prasanna Murali Krishna, is currently working as Associate Professor\&HOD,Dept of ECE, Dr.Samuel \\ George Institute of Engineering and Technology, Markapur, AP, India)
}

\begin{abstract}
This paper is focused on a new solution for congestion evasion in ad hoc multicast routing that referred as Congestion Evasion Multicast Routing short CEMR. CEMR is aimed at Congestion Evasion in Multicast Mobile Ad hoc routing protocol. The current MAC level routing strategy is independent which can work with any multicast routing protocol irrespective of tree or mesh structure. During the study of CEMR performance, the CEMR tested along with On-Demand Multicast Routing Protocol where simulation results proved that CEMR raises the performance of ODMRP in order of magnitude.
\end{abstract}

Keywords: multicast, on-demand routing, congestion control, ad hoc network, CEMR

\section{Introduction:}

Great numbers of routing protocols for Ad Hoc network are presented by classifying from many aspects. Protocols are of three types such as reactive protocols, proactive protocols and composite protocols that integrate the discovering process of the above ones. Depending on the structure of network topology, the protocols are divided in two types. They are plane ones and clustering ones. Depending on load balance mechanism, the protocols are grouped as single path ones and multi-path ones. A great number of routing protocols such as Dynamic Source Routing (DSR), Ad Hoc On-Demand Distance Vector Routing (AODV), Destination-Sequenced Distance-Vector Routing (DSDV), Temporally Ordered Routing Algorithm (TORA) and Zone Routing Protocol (ZRP), are identified but the possibility of using them and their efficiency remained doubt in view of the only min-hop metric as routing selection criterion. MANET has no difference between a host and a router, because all nodes are senders or receivers and also forwarders of traffic where all MANET members can deleted easily. Having high mobility nature, MANETs can be used in the environments, which require robust and reliable capacity like military battlefield, emergency rescue, vehicular communication, mining operations etc. For these applications, multicast is paramount and helpful in holding down network bandwidth and resources, as one message from one source can be sent to multiple receivers at a time. The main risk for multicast routing in MANETs is maintaining of robust capacity even in the condition of frequent, mainly high-speed agility and nodes outages. So mesh-based protocols builds a mesh for forwarding multicast packets for sending even in the presence of links breaking, and reaches robustness and reliability demands with path repetition owing to meshes on networks. Present multicast routing protocols for MANET is divided into two types: tree-based and mesh-based protocols. The tree based ones, i. e. MAODV (Multicast of Ad hoc On Demand Distance Vector) generally have tree-based schemes, unfits high-speed ad hoc networks. Common mesh-based multicast routing protocol is ODMRP (On-Demand Multicast Routing Protocol)[2], that uses the concept of forwarding group, builds multicast mesh that is done in soft state and acquires high performance [3, 4]. In [5], V. Kumar, et al. obtains comparative conclusions about MAODV and ODMRP based on the simulation results. Even though the performance of all multicast protocols degrade in terms of packet sending and group reliability as node mobility and traffic load augments, mesh-based protocol ODMRP do better job than tree-based protocol MAODV. ODMRP can bring forth decent robustness based on its mesh structure. MAODV performs less when compared to other protocols in packet delivery ratio and group reliability.

\section{Related Work}

Based on the researches on real life Ad Hoc network and references, it is seen that a prodigious of real life Ad Hoc networks works without following rules which are included in our theoretical analysis. In contrast to above, selfish nodes hinder the network process. The selfish nodes are intended in participating of the natural network information exchange procedure like routing discovery, routing maintenance and packets forwarding etc. The reason for selfishness of these nodes comes from the various advantages of various organizations who own the various groups of nodes. Because of the existence of selfish nodes, a few relay nodes remains as "hot spots" which leads to "death" due to power decrease resulting in total disabling of entire network. Prashant 
Dewan et al. said in [6] that, particular nodes in an Ad Hoc network might become antagonistic and thus refuse to cooperate with each other. In addition to, Ad hoc network posses semiautonomous nodes owned by different entities may not be distributed with common goal, and thus the nodes may not work together which is supposed to do. In [7], Buttayan et.al; presented "Nuglets" protocol for reducing the impact of nodes selfishness on entire network performance. The effectiveness gets "rewards" and in efficiency will be given "penalties"; In [8],"SPRITE" protocol is designed to control the selfishness by constructing a credit clearing service(CCS) server which provides a credit to every node in the network. The node selection depends on its credit for path. The above mentioned protocols focused on selfishness of nodes and how to overcome this selfishness. Whatever may so, implementation contains complexity in the channel fading, retransmission and collision etc. Thus these protocols remain incompetent.

\section{Congestion Evasion Multicast Routing (Cemr)}

The core point of CEMR is reliability transmission of every packet to every neighbor. The presented CEMR designed using a transmission window structure that influenced by IEEE 802.11 transmission structure, thus a brief operational overview of 802.11 transmissions is as follows.

\section{i. IEEE 802.11 Transmission Overview}

IEEE 802.11 used a collision evasion scheme including RTS/CTS/ACK control frames for unicast packets' transmission. The DCF of 802.11 shows the basic access method that mobile nodes uses for sharing wireless channel. The scheme combines CSMA with Collision Evasion (CSMA/CA) and acknowledgement (ACK). The mobile nodes based on need they can use the virtual carrier sense mechanism which provided RTS/CTS exchange for channel reservation and fragmentation of packets in situations. The CSMA/CA works in transmission of senses the channel. If the channel is free for a time equal to the DCF Inter Packet Space (DIFS) interval, the node transmits. If the channel is full of activity, the node enters a state of collision evasion and backs off from transmitting for a specified interval. In the collision evasion state, the node sensing the channel busy will suspend its back off timer, only resuming the back off countdown when the channel is again sensed free for a DIFS period. Common sequence of exchanges in 802.11 utilizing the virtual carrier sensing mechanism contains the source node first sensing the channel utilizing CSMA/CA. After the execution of CSMA/CA, RTS is transmitted by the source node, which follows responding of node with CTS, after responding the source node sends the data packet and subsequently with the conformation of destination node with an ACK to the source node. Receiving RTS, CTS or data packet is not real destination of any node but it should complete the data exchange is real destination of node. For broadcast packets, IEEE 802.11 nodes simply execute collision evasion and then transmit the data packet.

\section{ii. CEMR Transmission Window Structure}

CEMR, multicast node $n m$ need to manage two lists, List of Relay Nodes (LRN), which is hop level destinations, List of Packets Sent (LPS), transmission history. Receptions of frames (RTS/CTS/DATA/ACK/HELLO) are used by $\mathrm{nm}$ to maintain track of its targeted nodes. Every $\mathrm{nm}$ also maintains a LPS. The LPS contains copies of the frames which are already transmitted which may be need even later for retransmission. After receiving by neighbor a copy will be removed from the LPS. LPS size must be larger than targets number for any $\mathrm{nm}$. In addition to the LPS, there is possibility of storing yet to be transmitted packets which is called. Every target node maintains a list of Packets Received (LPR). LPR stores when a target node receives a new packet, it records the packet's sequence number in LPR. When a $\mathrm{nm}$ node transmits RTS to a destination node specifying a range of (from and to) sequence numbers, the destination node examines its LPR to determine whether it is missing any previous sequence numbers in the specified range. If so, the destination node replies with the missing sequence number in the CTS response.

Generally in CEMR, If an " $\mathrm{nm}$ " has to transmit a packet, it should first test the channel and then a collision evasion (CSMA/CA) step like that of 802.11. After the collision evasion step completion the channel becomes free, the $\mathrm{nm}$ sends RTS to its target picked from LRN that is particular range of sequence numbers which are already sent where the present sequence number is to be transmitted. All the process will be achieved by pulling the least sequence number from the LPS and defining it into the RTS packet with the present sequence number which is expected by the source node. After receiving the RTS, the determine target test its LPR and decides the needed sequence numbers. CTS response packet will react if the target node doesn't find precedent sequence number.

Similarly, Even It is current sequence id, the CTS response packet reacts. All further target nodes listening to the RTS will acquiesce long enough for the CTS/DATA/ACK transmission. Upon the receiving of the CTS, the " $n m$ "transmits the DATA (packet) according to the sequence number determined in the CTS packet. After receiving the DATA, the target node updates its LPR and answer with an ACK. Remaining neighboring nodes which receive the DATA updates their LPR. After receiving the ACK, if the DATA sent 
DATA is wrong but obtain from the buffer, the source node its process with the destination node with another RTS until the present DATA is sent from the queue. After transmission of the present DATA and recognized, the source node then buffers the packet and chooses the next neighbor in its NEIGHBOR LIST and repeats the whole process over again then the collision evasion step is neglected. In CEMR, ordered first strategy is used that picks a target node from LRN chronologically. During this process target nodes order changes depending on their current ingress ability status. The ordered first strategy sends packets and works comfortably. If, there are no packets for transmission of queue, the ordered first process will be stopped until next target in the LRN received all the broadcast DATA until there is a new packet to send. For preventing this, CEMR utilizes flag $c s$ that set to true and then next node in the LRN will be selected and the ordered first process repeats. In between if new sequence numbers joined then flag $c s$ will be set false then remaining targets are visited in the ordered first process without considering the current sequence numbers if any. Upon the completion of RTS to all targets in ordered first process if still no new sequence numbers are identified then ordered first process stops the RTS process till there a new packet is ready for transmission. If new sequence numbers is identified then the flag $c S$ sets wrong and ordered first process repeats.

\section{Congestion Evasion In Odmrp Using Cemr}

A few multicast routing protocols contains AMRoute[9], AMRIS[10], CAMP[11], multicast AODV[12], and the On-Demand Multicast Routing Protocol (ODMRP)[13, 14, 15]. ODMRP distracts multicast packets on a mesh in place of the traditional multicast tree. By using a mesh, ODMRP bring out excess to combat packet loss in ad hoc networks with mobility, collision, and channel noise. In regard to low traffic load, ODMRP does capably. Nevertheless, as traffic load augments, ODMRP continuously undergo from network congestion. Though this disadvantage is not limited to ODMRP it is wide spread among other multicast protocols. The present paper introduces a new MAC protocol, CEMR that allows reliable MAC broadcast in ad hoc networks. In addition to, by excess using CEMR, it is said that congestion control in ODMRP decreases network load when contention is high. This CEMR is not limited only to ODMRP but can apply even on other multicast protocols, like multicast AODV. ODMRP protocol is explained in the sub section I and ODMRP with our congestion evasion scheme CEMR is explained in the sub section ii where simulation results are provided in section 4. Subsequently, section 5 explains the conclusion of the paper.

\section{i. On-Demand Multicast Routing Protocol}

ODMRP creates a group-shared forwarding mesh for every group. Every source carries out periodic flood-response cycles creating multicast forwarding state without depending on present forwarding state. The frequent state discovery helps the protocol to find the present simple paths between every source and the multicast receivers and develops the boisterous protocol due to multiple forwarding paths may present between group members. Due to this ODMRP's packet send number of sources and receivers per multicast group augments and even sometimes increases mobility: the repeat forwarding state devises ODMRP's packet produces ability due to it behaves error correction, and does the protocol robust to mesh. Nevertheless, the frequent identification produces and great number of data transmissions identically augments network load.

Ever multicast source for a group G in ODMRP regularly moves the network with a JOIN QUERY packet that forwards by all nodes in the network. REFRESH INTERVAL, e. g., every 3 seconds send by this packet. Every multicast receiver reacts to this flow by delivering a JOIN REPLY packet that is forwarded in a simple path back to the multicast source that started the QUERY. Anterior of forwarding the packet, every node waits for JOIN AGGREGATION TIMEOUT, and mixes all JOIN REPLYs for the group received during this time into one JOIN REPLY. Every node that forwards the REPLY packet generates (or refreshes) forwarding state for group G. Every node with forwarding state for $\mathrm{G}$ forwards every data packet delivered by a multicast source for G. A data packet use the simplest paths to the multicast receivers within the forwarding mesh, it may even forwarded to other sources of the group who are group members. Forwarding state is ceased after a multiple of regular breaks to assure that in the event that some number of forwarding nodes' multicast state is not refreshed due to packet loss, the forwarding state generated from a earlier flood is also authenticated. This mechanism develops the boisterous protocol, where many overlapping trees will be activated in the network parallel; everything is produced finally by JOIN QUERY flood [16].

\section{A. Route discovery}

In CEMR, route finds is started and managed by the source. When the source contains packets for transmission for a certain multicast group, the source first decided if there is a route to the group. If a route is not present, CEMR tries to create one through the route finding process. The route finding process is equal to on-demand unicast routing protocols like AODV[17] and DSR[18]. Route discovery process has two steps. The first round is a request round and a reply round. 


\section{a. Route Request}

In the request round, the source moves the network with a member advertisement packet with the data piggybacked which is named as JOIN QUERY. These JOIN QUERY packets regularly broadcast to the total network to refresh membership information and recreate new multicast routes. Once receiving a “ JOIN QUERY" that is not a replica of earlier, a node inserts or updates in its ROUTING TABLE the upstream node indicates as the next node to the source node. The ROUTING TABLE can also be utilized even in a JOIN REPLY depending on need of the source during the reply round, called as backward learning [19].

\section{b. Route Reply}

After reaching a non-duplicate JOIN QUERY to multicast member the reply round begin. In the reply round, the multicast member generates and broadcasts JOIN REPLY packet to the network with the address of the node the member receives the JOIN QUERY from stamped in the JOIN REPLY. After receiving the JOIN REPLY, a node decides if its address is stamped in the JOIN REPLY. If so, the node knows it is on the path to the source and set the path FORWARDING_GROUP_FLAG and be a part of the forwarding group. After that, the node resends JOIN REPLY with the upstream node address to the source stamped in the JOIN REPLY. The upstream node address is got from the ROUTE TABLE via rearward learning. This procedure goes in anticipation of the JOIN REPLY meets the source. The source obtains JOIN REPLY, a mesh of nodes, or forwarding groups, is formed and packets can be sent to the members.

\section{c. Route maintenance}

ODMRP manages the group by regular broadcasting JOIN QUERY to the network and receiving JOIN REPLY. The regular broadcast of JOIN QUERY updates the forwarding group nodes and takes membership fluctuations.

\section{ii. ODMRP with Congestion evasion}

To accomplish congestion evasion, CEMR is utilized as the underlying MAC layer. CEMR is needed because ODMRP broadcasts data packets to all neighbors in spite of sending them point-to-point to choose individual neighbors, as causally done by multicast protocols. The underlying MAC protocol utilized for broadcast, CSMA avoiding ACK. CSMA, the queue length will denote perfect measure of congestion. Broadcast packets are sending "blindly". If the packet is not reached because of receive-buffer excess flow or channel congestion. it is stopped and no retransmission is done. Accordingly, even in presence of congestion, the queue length is little. In opposite to, the version of the IEEE 802.11 protocol utilized in unicast, point-topoint transmissions is filled with RTS and CTS control packets and ACKs. It is used against receive-buffer overflow and hidden terminals, and thus supplies perfect congestion feedback. This unicast version accordingly is not so attractive for multicast applications because it cannot misuse "broadcast advantage" of the wireless channel, and needs an individual transmission to every multicast member. Hence, CEMR is required to perfect description of the network state via queue lengths as CEMR supply reliable delivery of packets that are broadcasted in the context of multicast.

\section{A. Congestion Evasion:}

CEMR effectively eliminates the congestion by adapting to ordered first sequence to cast the packet all target nodes in broadcast manner. Here the CEMR process surveyed with an example.

The concept of CEMR is explained with an example. First of all we take a tree based multicasting or mesh based multicasting. After the path discovery process, let consider a multicast group such that a node $n 5$ desires to multicast packets to nodes $n 1, n 4, n 6, n 9$. At the stage of transmitting first packet Node n5 first decides a target node $\mathrm{n} 1$ from LRN and sends RTS with sequence numbers ranging from $\mathrm{p} 0$ to $\mathrm{p} 0$ since no DATA frames have yet been sent. Node $\mathrm{n} 1$, upon receiving the RTS packet, responds with sequence number p0 in the CTS packet. Nodes n6, n9, and n4, after receiving the RTS packet, gives for the CTS/DATA/ACK interchange between node $n 5$ and $n 1$. After receiving the CTS packet, node n5 multicasts DATA with sequence number 0 in broadcasting manner. Node 1, after receiving DATA, updates its LPR and responds with an ACK. For explanation requirement, a node n6 did not receive the DATA (possibly due to interference from neighboring nodes) while node $\mathrm{n} 9$ and $\mathrm{n} 4$ received the DATA perfectly. Hence, node $\mathrm{n} 9$ and $\mathrm{n} 4$ also update their RF. After receiving the ACK, node n5 copies the DATA that was delivered into the LPS and goes on to choose nodes from LRN in ordered first form. If we imagine that node n6 chosen in order as immediate neighbor for transmission after executing the collision evasion round, node $n 5$ delivers RTS with sequence number range p0 to $\mathrm{p} 1$. Upon receiving the RTS, node $\mathrm{n} 6$ looks its LPR and noticed that packet $\mathrm{p} 0$ haven't received. Node n6 then delivers CTS desired sequence number p0. Node n5, after receiving the CTS, gets the DATA with sequence number p0 from the LPS and transmits the DATA. After receiving the DATA, node n6 updates its LPR and responds with an ACK. Upon receiving the ACK, node n5 delivers RTS repeatedly with sequence 
number range $\mathrm{p} 0$ to $\mathrm{p} 1$ since the most recent DATA will not been sent. Node $\mathrm{n} 6$, after receiving the RTS then delivers CTS with sequence number 1 after checking its LPR. Node n5, upon receiving the CTS, sends the DATA with sequence number p1. Node n6, after receiving the DATA, response with an ACK again, for explanation process, let's say nodes $\mathrm{n} 1, \mathrm{n} 9$ and $\mathrm{n} 4$ receive the DATA and update their respective LPR. Node $\mathrm{n} 5$, after receiving the ACK, buffers the DATA in LPS and selects node $\mathrm{n} 9$ as its immediate neighbor. After the collision evasion round, node $\mathrm{n} 5$ transmits RTS with sequence number range $\mathrm{p} 0$ to $\mathrm{p} 2$. After receiving the RTS, node $\mathrm{n} 9$ inquiry its received sequence number list and delivers CTS requesting sequence number $\mathrm{p} 2$ (since $\mathrm{p} 0$ and $\mathrm{p} 1$ were successfully received before). Node $\mathrm{n} 5$, after receiving CTS, transmits DATA with sequence number p2. Node $\mathrm{n}$ 9, after receiving DATA, transmits ACK and updates its LPR. Node n5, after receiving ACK, buffers the DATA in LPS, choose node $n 4$ as it's immediate neighbor to transmit to, and the process starts again. In this process if node $\mathrm{n} 5$ found that no data with new sequence numbers available, then it set flag cs true and goes on delivering RTS with sequence range already delivered and cached in LPS to nodes in LRN in ordered first form. After sending RTS to all the nodes in LRN, checks for data. If still no data with new sequence numbers then this process stops till it discovers data with new sequence numbers. If data is exposed with new sequence number then flag $c s$ sets to wrong and promotes multicast process.

\section{B. CEMR Algorithm}

Description of the notations

1. $n m \leftarrow$ Node participating in multicasting

2. $n u \leftarrow$ Node participating in one of the unicasting path of $n m$

3. $T N L \leftarrow$ Target Node List

4. $b p_{n m} \leftarrow$ Buffer of Packets to multicast at $n m$

5. $\quad F S_{n m} \leftarrow$ Buffer of Frames already sent by $n m$

6. $F R_{t n} \leftarrow$ Buffer of Packets Received by target node $t n$ that listed in $T N L$

7. $c S \leftarrow$ Boolean flag

Input:

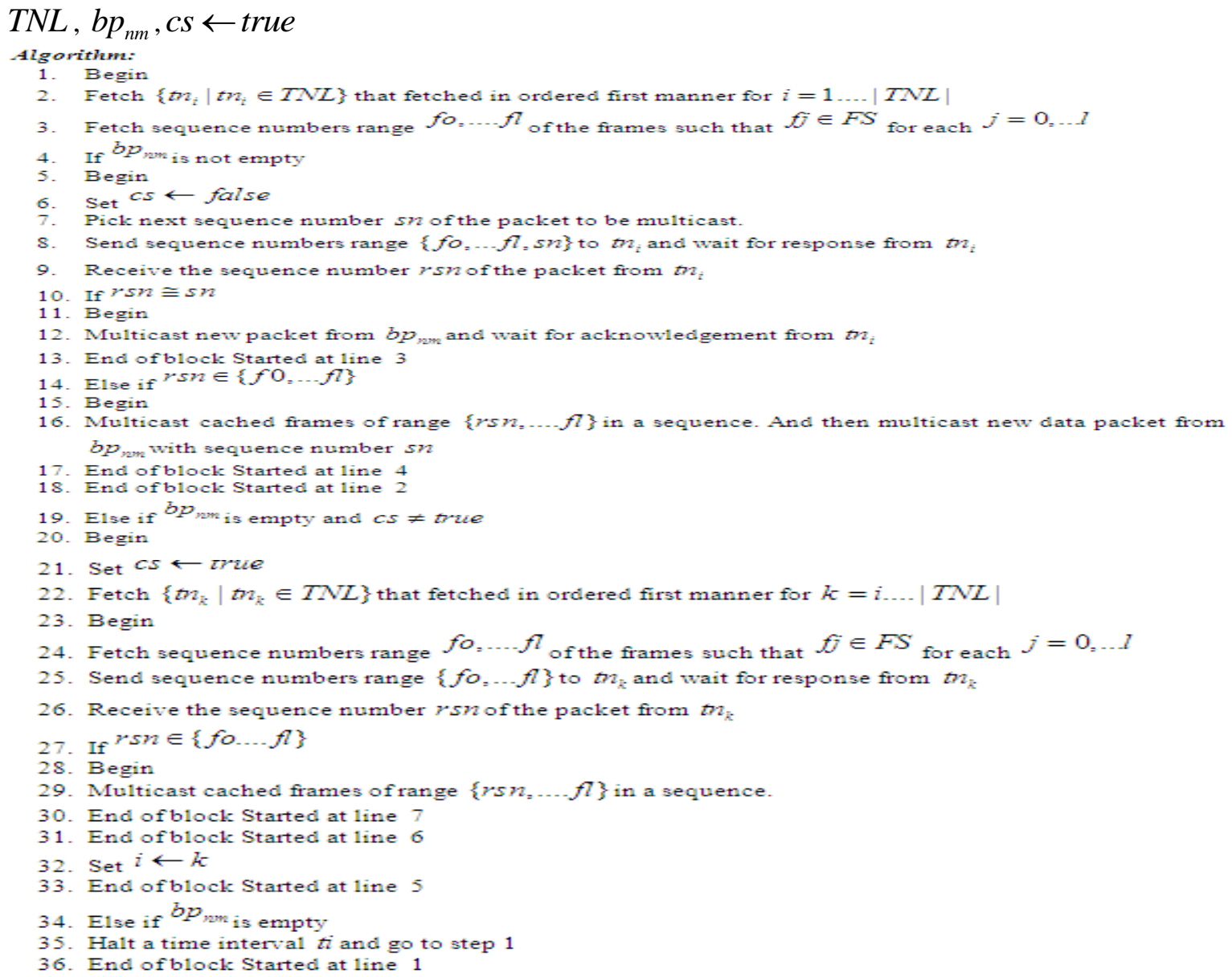


In step 12, 16 and 29 all nodes of list $T N L$ also receives those frames and according their respective $F R$ status they update $F R$, that is if the nodes not found that packet in their respective $F R$ then updates otherwise discards.

In step 12 and 16, if acknowledgement received from target node $t n_{i}$ then the node $n m$ updates it's $F S_{n m}$ by adding new sequence number to $F S_{n m}$

\section{Simulations And Results Discussion}

NS 2 is used in doing experiments. We create a simulation network with hops under mobility and count of 50 to 200. The simulation parameters explained in table 1. Authentication ensures that the buffer is properly allocated to valid packets. The simulation model aims in comparing ODMRP with CEMR and ODMRP. The performance examines of these two brings out against to the metrics displayed in the given table:

\begin{tabular}{|l|l|}
\hline Number of nodes Range & 50 to 200 \\
\hline Dimensions of space & $1500 \mathrm{~m} \times 300 \mathrm{~m}$ \\
\hline Nominal radio range & $250 \mathrm{~m}$ \\
\hline Source-destination pairs & 20 \\
\hline Source data pattern (each) & 4 packets/second \\
\hline Application data payload size & 512 bytes/packet \\
\hline Total application data load range & 128 to $512 \mathrm{kbps}$ \\
\hline Raw physical link bandwidth & $2 \mathrm{Mbps}$ \\
\hline Initial ROUTE REQUEST timeout & 2 seconds \\
\hline Maximum ROUTE REQUEST timeout & 40 seconds \\
\hline Cache size & 32 routes \\
\hline Cache replacement policy & FIFO \\
\hline Hash length & 80 bits \\
\hline certificate life time & $2 \mathrm{sec}$ \\
\hline
\end{tabular}

Table1: The parameters used in simulation experiments

The metrics to verify the performance of the present protocol as follows:

$>$ Data packet delivery ratio: Data packet delivery ratio is calculated as the ratio between the number of data packets that are delivering by the source and the number of data packets that are received by the sink.

$>$ PACKET DELIVERY FRACTION: It is the ratio of data packets send to the destinations to those created by the sources. The PDF says about the performance of a protocol that how successfully the packets have been send. Higher the value produces the better results.

$>$ AVERAGE END TO END DELAY: Average end-to-end delay is an average end-to-end delay of data packets. Buffering during route detection latency, queuing at interface queue, retransmission delays at the MAC and transfer times, may cause this delay. Once the time variation between packets sent and received was recorded, dividing the total time variation over the total number of CBR packets received provided the average end-to-end delay for the received packets. Lower the end to end delay enhanced is the performance of the protocol.

$>$ Packet Loss: It is defined as the variation between the number of packets sent by the source and received by the sink. In our results we have intended packet loss at network layer as well as MAC layer. The routing protocol ahead the packet to destination if a valid route is known; otherwise it is buffered until a route is obtainable. There are two cases when a packet is dropped: the buffer is full when the packet needs to be buffered and the time exceeds the limit when packet has been buffered. Lower is the packet loss enhanced is the performance of the protocol.

$>$ ROUTING OVERHEAD: Routing overhead is calculated at the MAC layer which is defined as the ratio of total number of routing packets to data packets.

Figure 1(a) displays the Packet Delivery Ratio (PDR) for ODMRP [15] and ODMRP with CEMR. Depending on the results it is clear that CEMR reduces the loss of PDR that observed in ODMRP [15]. The approximate PDR loss recovered by CEMR over [15] is $14.471 \%$, this is an average of all pauses. The minimum individual recovery observed is $5.91 \%$ and maximum is $30.345 \%$.

The packet delivery fraction (PDF) is denoted as: 
$P^{\prime}=\sum_{f=1}^{e} \frac{R_{f}}{N_{f}}$

$P=\frac{1}{c} * P^{\prime}$

- $\quad P$ is the fraction of successfully delivered packets,

- $c$ is the total number of flow or connections,

- $f$ is the unique flow id serving as index,

- $R_{f}$ is the total of packets obtained from flow $f$

- $\quad N_{f}$ Is the count of packets transmitted to flow $f$

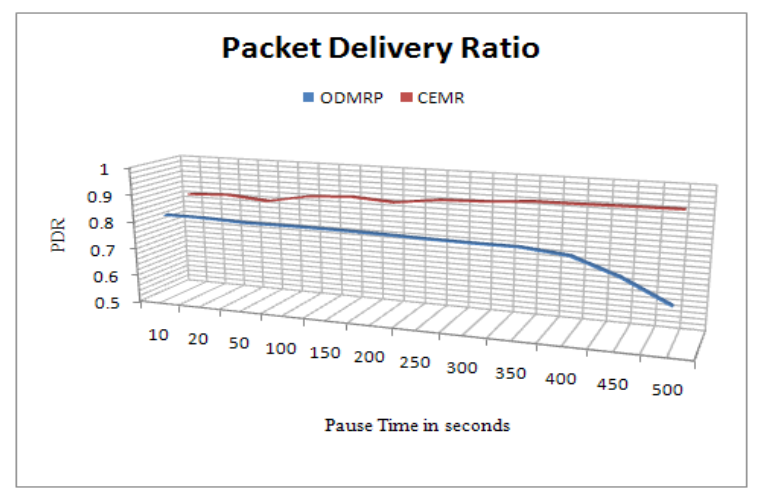

(a) Packet delivery ratio

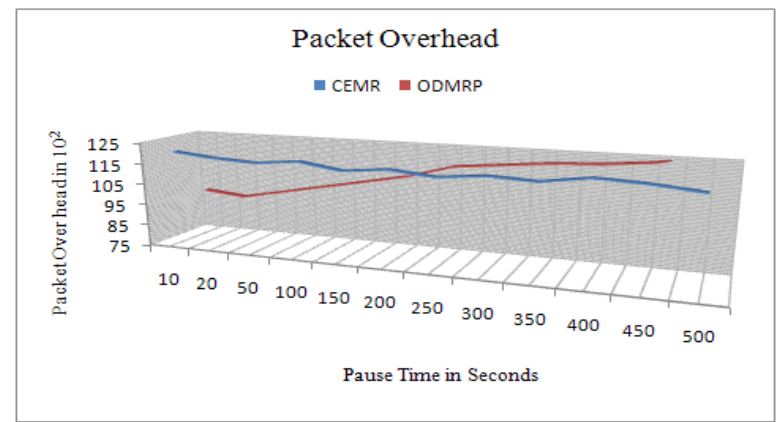

(b) Packet overhead comparison report

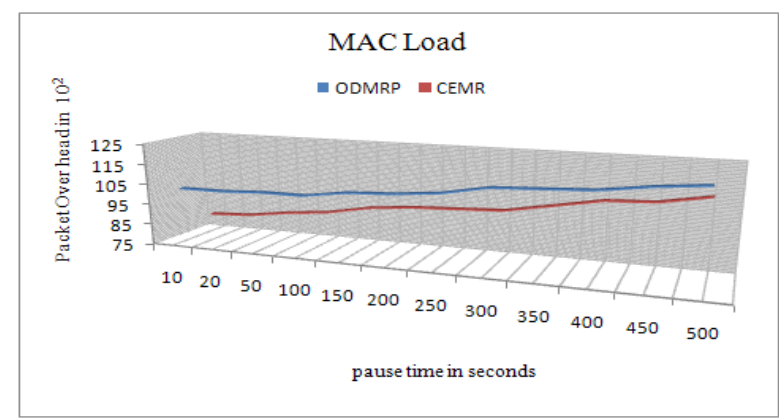

(c) Mac load comparison

Fig 1: Comparison of performance metric values between ODMRP with CEMR and ODMRP.

Figure 1(b) affirms that ODMRP with CEMR have stable packet overhead over ODMRP [15] where the magnitude growth in packet overhead in different pause intervals. Because of congestion evasion routing mechanism of CEMR this benefit is possible. The average Packet overhead observed for 12 intervals in ODMRP with CEMR is 117. 9 more than packet overhead observed for 12 intervals in ODMRP. But the average growth of the packet over head in ODMRP is $26.36 \%$, but in the case of ODMRP with CEMR, the average growth in packet over head is 3. 34\%. This advantage of ODMRP with CEMR over ODMRP happens due to the collision and congestion evasion strategy introduced in CEMR. 
The advantage of ODMRP with CEMR over ODMRP in MAC load overhead control is shown in the Figure 1(c), . The average growth in MAC load over head in ODMRP with CEMR is $14.32 \%$ is almost equal to MAC load overhead in ODMRP, which are 14. 17\%, this resulted due to multicasting of the packets in CEMR to all target nodes unlike in ODMRP, a unicasting packet.

\section{Conclusion:}

This paper expatiate a MAC level multicast routing algorithm called "Congestion Evasion Multicast Routing" i. e. CEMR. The projected routing approach aims at evasion of congestion at group levels formed in multicast route discovery. This protocol derives an algorithm that transmits the data in multicast manner at group level unlike other multicast protocols, concentrating of data transmission in a sequence to every targeted node. Being independent, the CEMR works with group of either tree or mesh. The present mentioned CEMR is tested by associating with ODMRP where the simulation results indicated that the CEMR improves the PDR and reduces the Packet overhead of ODMRP in order of magnitude. It is further planned to develop an extension to CEMR which can even control the congestion besides avoiding congestion.

\section{References:}

[1] G. Krishna, "Routing protocols in mobile ad-hoc networks," pp. 1-30, 2006 http://www.cs.umu.se/ education/examina/Rapporter/Krish naGorantala.pdf

[2] L. Sung-Ju, S. William, G. Mario, "On-demand multicast routing protocol in multihop wireless mobile networks," Mobile Networks and Applications, 2001.

[3] S. J. Lee, W. Su, J. Hsu, M. Gerla, and R. Bagrodia, "A performance comparison study of ad hoc wireless multicast protocols," Proceedings of the IEEE INFOCOM'00, 2000

[4] P. Madhan, J. James, K. Murugan, V. Ramachandran, “A Comparative and Performance Study of On Demand Multicast Routing Protocols for Ad Hoc Networks," 9th International Conference on High Performance Computing (HiPC), 2002.

[5] V. Kumar, O. Katia and T. Gene, "Exploring mesh- and tree based multicast routing protocols for MANETs," IEEE Transactions on Mobile Computing, vol. 5, pp. 28-42, 2006

[6] Prashant Dewan, Partha Dasgupta and Amiya Bhattacharya; "On Using Reputations in Ad hoc Networks to Counter Malicious Nodes" Tenth International Conference on Parallel and Distributed Systems, 2004; ICPADS 2004. Proceedings; July 2004

[7] L. Butty'an and J.-P. Hubaux. Enforcing Service Availability in Mobile Adhoc WANs, In ACM international symposium on Mobile ad hoc networking and computing, pages 87-96, Boston, Massachusetts, 2000. ACM Press

[8] S. Zhong, J. Chen, and R. Yang Sprite: A Simple, Cheat-proof, Credit-based System for Mobile Ad-hoc Networks. In IEEE INFOCOM, San Francisco, USA, 2002. IEEE Press.

[9]. E. Bommaiah, M. Liu, A. McAuley, and R. Talpade, “AMRoute: Ad-hoc Multicast Routing Protocol,” Internet- Draft, drafttalpade-manet-amroute-00.txt, Aug. 1998, Work in progress.

[10] C.W. Wu, Y.C. Tay, and C.-K. Toh, “Ad hoc Multicast Routing Protocol utilizing Increasing id-numberS (AMRIS) Funcational Specification," Internet-Draft, draft-ietf-manet-amris-spec-00.txt, Nov. 1998, Work in progress

[11] J.J. Garcia-Luna-Aceves and E.L. Madruga, "The Core-Assisted Mesh Protocol," IEEE Journal on Selected Areas in Communications, vol. 17, no. 8, Aug. 1999, pp. 1380-1394.

[12] E. M. Royer and C. E. Perkins, "Multicast Operation of the Ad-hoc On-Demand Distance Vector Routing Protocol," Proceedings of ACM/IEEE MOBICOM'99, Seattle, WA, Aug. 1999.

[13] S.-J. Lee, M. Gerla, and C.-C Chiang, “On-Demand Multicast Routing Protocol,” Proceedings of IEEE WCNC'99, New Orleans, LA, Sep. 1999, pp. 1298-1302.

[14] S.-J. Lee, W. Su, J. Hsu, M. Gerla, and R. Bagrodia, "A Performance Comparison Study of Ad Hoc Wireless Multicast Protocols," Proceedings of IEEE INFOCOM2000, Tel Aviv, Israel, Mar. 2000.

[15] S.-J. Lee, W. Su, and M. Gerla, Internet Draft, draft-ietf-manet-odmrp-02.txt, Jan. 2000

[16] J. Haartsen, M. Naghshineh, J. Inouye, O.J. Joeressen, and W. Allen, "Bluetooth: Vision, Goals, and Architecture," ACM SIGMOBILE Mobile Computing and Communications Review, vol. 2, no. 4, Oct. 1998, pp. 38-45.

[17] C. E. Perkins and E. M. Royer, “Ad-Hoc On Demand Distance Vector Routing," Proceedings of the 2nd IEEE Workshop on Mobile Computing Systems and Applications (WMCSA), New Orleans, LA, Feb. 1999.

[18] D. B. Johnson and D. A. Maltz, "Dynamic Source Routing in Ad Hoc Wireless Networks," Mobile Computing, edited by Tomasz Imielinski and Hank Korth, Kluwer Academic Pusblishers, 1996.

[19] S. Keshav, "An Engineering Approach to Computer Networking: ATM Networks, the Internet, and the Telephone Network", Addison-Wesley, Menlo Park, California, 1997. 